\title{
Equilibrium Mode Distribution and Steady State Distribution in Step-Index Glass Optical Fibers
}

\author{
S. Savović $\bar{c}^{a, b, *}$, A. Djordjevich ${ }^{a}$, B. DrluaČA ${ }^{b}$ And A. Simović ${ }^{b}$ \\ ${ }^{a}$ City University of Hong Kong, 83 Tat Chee Avenue, Hong Kong, China \\ ${ }^{b}$ Faculty of Science, R. Domanovica 12, 34000 Kragujevac, Serbia
}

\begin{abstract}
Using the power flow equation, we have examined mode coupling in a step-index multimode glass optical fiber. As a result, the coupling length at which the equilibrium mode distribution is achieved and the length of fiber required for achieving the steady-state mode distribution are obtained. These lengths are much longer for glass fiber than they are for plastic optical fibers. Our results are in good agreement with experimental results reported earlier.
\end{abstract}

PACS numbers: 42.81.-i, 42.81.Qb, 42.81.Uv

\section{Introduction}

For decades, glass optical fibers (GOFs) have been the preferred transmission medium in high-capacity communications networks and long-distance communications systems. In contrast, plastic optical fibers (POFs) are usually considered for short data links. Local networking with POFs benefits from the rapid (less laborious) interconnectivity with low precision and cost components as POFs couple light efficiently due to their large diameter $(\approx 1 \mathrm{~mm})$ and high numerical aperture. However, POF performance is clearly attenuation limited. A typical attenuation level for step-index (SI) POFs is $\approx 100 \mathrm{~dB} / \mathrm{km}$ - compared with $\approx 0.5 \mathrm{~dB} / \mathrm{km}$ for SI GOFs [1]. This limits POF data links to lengths shorter than $100 \mathrm{~m}$. Typically, optical implementations of long-distance communications systems (greater than $1 \mathrm{~km}$ ) employ GOF.

Transmission characteristics of step-index optical fibers depend strongly upon the differential mode attenuation and the rate of mode coupling. The latter represents power transfer from lower to higher order modes caused by fiber impurities and inhomogeneities introduced during the fiber manufacturing process (such as microscopic bends, irregularity of the core-cladding boundary and refractive index distribution fluctuations). In the absence of these intrinsic perturbation effects, light launched at a specific angle with respect to the fiber axis forms a sharply defined ring radiation-pattern at the output fiber end. Due to mode coupling, the boundaries of the ring become blurred at the end of longer fibers. This fussiness increases with fiber length. As the ring-pattern evolves gradually, it eventually takes the form of a disk covering the entire fiber cross-section in fibers longer than the "coupling length" $L_{\mathrm{c}}$. The "equilibrium mode distribution" (EMD) exists beyond the coupling length $L_{\mathrm{c}}$ of the fiber. It is characterized by the absence of rings re-

* corresponding author; e-mail: savovic@kg.ac.rs gardless of the launch conditions, even though the disk pattern may have different light distributions across it depending on the launch conditions. EMD indicates a substantially complete mode coupling and is of critical importance when measuring characteristics of multimode optical fibers (linear attenuation, bandwidth, etc.). Indeed, measurement of these characteristics would only be considered as meaningful if performed at the EMD condition when it is possible to assign to a fiber a unique value of loss per unit length [2]. At distance $z_{s}\left(z_{s}>L_{\mathrm{c}}\right)$ from the input fiber end, all individual disk patterns corresponding to different launch angles take the same light-distribution across the fiber-section and the "steady state distribution" (SSD) is achieved. SSD indicates the completion of the mode coupling process and the independence of the output light distribution from launch conditions.

Output angular power distribution in the near and far fields of an optical fiber end has been studied extensively. Work has been reported using geometric optics (ray approximation) to investigate mode coupling and predict output-field patterns $[3,4]$. By employing the power flow equation [5-9] as well as the Fokker-Planck and Langevin equations [10], these patterns have been predicted as a function of the launch conditions and fiber length. In this paper, our solution of the power flow equation determines the coupling length $L_{\mathrm{c}}$ for achieving the EMD, the transition states along $L_{\mathrm{c}}$, as well as the length $z_{s}$ for achieving the SSD. To allow comparisons, results are illustrated on the case of the SI GOF investigated earlier by Jeunhomme et al. [11].

\section{Power flow equation}

Gloge's power flow equation is $[6]$ :

$$
\frac{\partial P(\theta, z)}{\partial z}=-\alpha(\theta) P(\theta, z)+\frac{D}{\theta} \frac{\partial}{\partial \theta}\left(\theta \frac{\partial P(\theta, z)}{\partial \theta}\right),
$$

where $P(\theta, z)$ is the angular power distribution, $z$ is distance from the input end of the fiber, $\theta$ is the prop- 
agation angle with respect to the core axis, $D$ is the coupling coefficient assumed constant $[5,7]$ and $\alpha(\theta)$ is the modal attenuation. The boundary conditions are $P\left(\theta_{\mathrm{c}}, z\right)=0$, where $\theta_{\mathrm{c}}$ is the critical angle of the fiber, and $D(\partial P / \partial \theta)=0$ at $\theta=0$. Condition $P\left(\theta_{\mathrm{c}}, z\right)=0$ implies that modes with infinitely high loss do not carry power. Condition $D(\partial P / \partial \theta)=0$ at $\theta=0$ indicates that the coupling is limited to the modes propagating with $\theta>0$. Except near cutoff, the attenuation remains uniform $\alpha(\theta)=\alpha_{0}$ throughout the region of guided modes $0 \leq \theta \leq \theta_{\mathrm{c}}$ [7] (it appears in the solution as the multiplication factor $\exp \left(-\alpha_{0} z\right)$ that also does not depend on $\left.\theta\right)$. Therefore, $\alpha(\theta)$ need not be accounted for when solving (1) for mode coupling, and this equation reduces to [8]:

$$
\frac{\partial P(\theta, z)}{\partial z}=\frac{D}{\theta} \frac{\partial P(\theta, z)}{\partial \theta}+D \frac{\partial^{2} P(\theta, z)}{\partial \theta^{2}} .
$$

The solution of Eq. (2) for the steady-state power distribution is given by [7]:

$$
P(\theta, z)=J_{0}\left(2.405 \frac{\theta}{\theta_{\mathrm{c}}}\right) \exp \left(-\gamma_{0} z\right),
$$

where $J_{0}$ is the Bessel function of the first kind and zero order and $\gamma_{0}\left[\mathrm{~m}^{-1}\right]=2.405^{2} \mathrm{D} / \theta_{\mathrm{c}}^{2}$ is the attenuation coefficient. We used this solution to test our numerical results for the case of the fiber length at which the power distribution becomes independent of the launch conditions.

In order to obtain numerical solution of the power flow Eq. (2) we have used the explicit finite-difference method (EFDM) to investigate mode coupling in short step-index plastic optical fibers (length up to hundred meters) $[8,12,13]$. We now employe EFDM, in our knowledge for the first time, for solving the power flow equation in much longer (kilometers in length) step-index glass optical fiber. This should also be a test of accuracy and efficiency of the EFDM for such a long fiber.

To start the calculations, we used Gaussian launch-beam distribution of the form

$$
P(\theta, z)=\exp \left(-\frac{\left(\theta-\theta_{0}\right)}{2 \sigma^{2}}\right),
$$

with $0 \leq \theta \leq \theta_{c}$, where $\theta_{0}$ is the mean value of the incidence angle distribution, with the full width at half maximum FWHM $=2.355 \sigma$ ( $\sigma$ is standard deviation $)$. This distribution is suitable both for LED and laser beams.

\section{Numerical results}

In this paper, we analyze mode coupling in the CGW-CGE-68 step-index multimode glass optical fiber used in the experiment reported earlier [11]. This fiber's critical angle is $\theta_{\mathrm{c}}=7.26^{\circ}$ (measured inside the fiber), i.e. $\theta_{\mathrm{c}}=$ $10.6^{\circ}$ (measured in air). Value of coupling coefficient of $D=7.9 \times 10^{-7} \mathrm{rad}^{2} / \mathrm{m}$ has been reported for this fiber [11] (it is required in Eq. (1)) — which we have adopted in this work.

Our numerical solution of the power flow equation gives the evolution of the normalized output power distribution with fiber length $z$ as $z$ is incremented from zero until achieving the SSD. The situation is shown in Fig. 1 for selected four such $z$ values, each for three different input angles $\theta_{0}=0,3$ and $6^{\circ}$ (measured inside the fiber). We selected Gaussian launch beam distribution with FWHM $=1.2^{\circ}$ by setting $\sigma=0.5095^{\circ}$ in Eq. (4). We used the step lengths of $\Delta \theta=0.05^{\circ}$ and $\Delta z=0.0005 \mathrm{~m}$ in order to achieve stability of our finite difference scheme $[8,14]$.

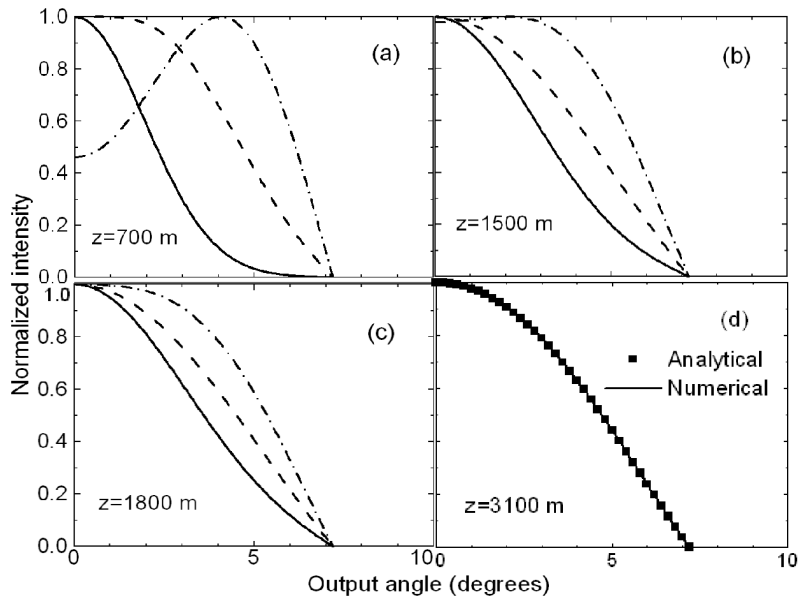

Fig. 1. Normalized output angular power distribution at different locations along the SI GOF calculated for three Gaussian input angles $\theta_{0}=0^{\circ}$ solid line), $3^{\circ}$ (dashed line) and $6^{\circ}$ (dash-dotted line) with FWHM $=$ $1.2^{\circ}$ for: (a) $z=700 \mathrm{~m}$, (b) $z=1500 \mathrm{~m}$, (c) $z=1800 \mathrm{~m}$, and (d) $z=3100 \mathrm{~m}$ (filled squares represent the analytical steady-state solution).

One can observe from radiation patterns in the fiber of shorter length $z=700 \mathrm{~m}$ in Fig. 1a that the coupling is stronger for the low-order modes: the Gaussian beam launched at $3^{\circ}$ has already shifted to $\theta=0^{\circ}$. Coupling of higher order modes can be observed better only after more substantial fiber lengths (Fig. 1b). It is not until the fiber's coupling length $L_{\mathrm{c}}=1800 \mathrm{~m}$ that all the mode-distributions shift their mid-points to zero degrees (from their respective initial values at the input fiber end), producing the EMD in Fig. 1c. The coupling continues further along the fiber beyond the $L_{\mathrm{c}}$ mark until all distributions' widths equalize and SSD is reached at the length of $z_{s}=3100 \mathrm{~m}$, Fig. 1d. Figure 1d shows normalized curves of the output angular power distribution obtained by the power flow equation by the EFDM (solid curve) as well as the steady state analytical solution of Eq. (2) (squares), where $\gamma_{0}=2.86 \times 10^{-4} \mathrm{~m}^{-1}$. The two solutions are in good agreement with the relative error below the $0.95 \%$. It is important to note here that although the total integration fiber length (length for achieving SSD) in this case is of the order of kilometers, which is much longer than the length for achieving SSD in plastic optical fibers (typically up to hundred meters), the accumulated truncation errors of the applied numerical procedure is not too much larger if compared to the case of short plastic optical fibers where relative error is usually up to $0.2 \%[8,12,13]$. Thus, we conclude that EFDM is efficient and accurate for solving the 
power flow equation, both in the case of short (plastic) and very long (glass) optical fibers. Our numerical result for $z_{s}$ is in good agreement with the experimental result by Jeunhomme et al. [11]. who obtained, by varying the launch beam angle, that SSD is achieved in $3000 \mathrm{~m}$ long SI GOF (Jeunhomme's experimental steady state output-field pattern is shown in Fig. 2).

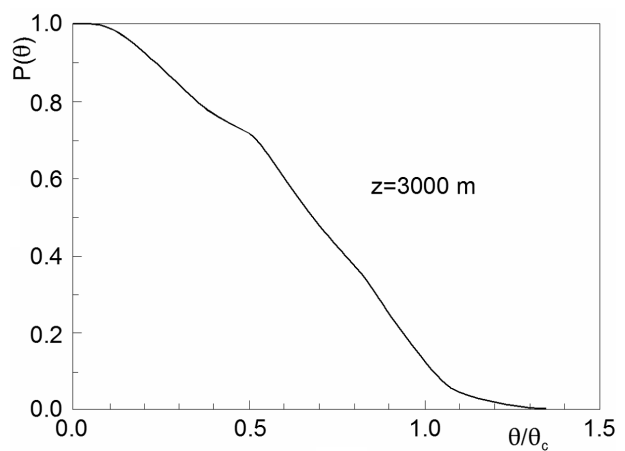

Fig. 2. Experimentally determined [11] normalized steady state output angular power distribution for $\mathrm{He}-\mathrm{Ne}$ laser launch beam at fiber length of $3000 \mathrm{~m}$ (reconstructed).

In summary, our numerical results show a much longer coupling length $\left(L_{\mathrm{c}}=1800 \mathrm{~m}\right)$ in SI GOF than in SI POFs (typically $L_{\mathrm{c}} \cong 15$ to $35 \mathrm{~m}[13,14]$ ). Similarly, the length for achieving the SSD is also much longer in the SI GOF $\left(z_{s}=3100 \mathrm{~m}\right)$ than in SI POFs (usually up to one hundred meters). This is attributed to the weaker intrinsic perturbation effects in SI GOFs.

\section{Conclusions}

Using the power flow equation, we have examined the state of mode coupling in a long step-index glass optical fiber by observing, for different launch angles, the output angular power distribution as it varied with the fiber length. For the CGW-CGE-68 fiber, we have obtained that the coupling length for achieving the equilibrium mode distribution is $1800 \mathrm{~m}$ and the length for achieving the steady state power distribution is $3100 \mathrm{~m}$. This is much longer than for plastic optical fibers with typi- cal coupling lengths from 15 to $35 \mathrm{~m}$ [13]. On a separate note, the explicit finite difference method applied in our knowledge for the first time to solve the power flow equation in a kilometers long fiber has turned out to be efficient and accurate.

\section{Acknowledgments}

The work described in this paper was supported by a grant from City University of Hong Kong (project No. 7002313) and by a grant from Serbian Ministry of Science (project No. 141023).

\section{References}

[1] W. Daum, J. Krauser, P.E. Zamzow, O. Ziemann, POF-Polymer Optical Fibers for Data Communications, Springer, Berlin 2002.

[2] J. Dugas, G. Maurel, Appl. Opt. 31, 5069 (1992).

[3] M. Eve, J.H. Hannay, Opt. Quantum Electron. 8, 503 (1976).

[4] J. Arrue, G. Aldabaldetreku, G. Durana, J. Zubía, I. Garcés, F. Jiménez, J. Lightwave Technol. 23, 1253 (2005).

[5] W.A. Gambling, D.N. Payne, H. Matsumura, Appl. Opt. 14, 1538 (1975).

[6] D. Gloge, Bell Syst. Tech. J. 51, 1767 (1972).

[7] M. Rousseau, L. Jeunhomme, IEEE Trans. Microwave Theory Tech. 25, 577 (1977).

[8] A. Djordjevich, S. Savović, IEEE Photon. Technol. Lett. 12, 1489 (2000).

[9] S. Savović, A. Djordjevich, Appl. Opt. 41, 7588 (2002).

[10] S. Savović, A. Djordjevich, Appl. Opt. 41, 2826 (2002).

[11] L. Jeunhomme, M. Fraise, J.P. Pocholle, Appl. Opt. 15, 3040 (1976).

[12] S. Savović, A. Djordjevich, Appl. Opt. 43, 5542 (2004).

[13] S. Savović, A. Djordjevich, Appl. Opt. 45, 6775 (2006).

[14] G. Jiang, R.F. Shi, A.F. Garito, IEEE Photon. Technol. Lett. 9, 1128 (1997). 